Objectives: To assess the efficiency and utilisation of virtual and telephone clinics in a general rheumatology department.

Methods: Data was collected from electronic (Cerner) patient records on 240 patients who had a virtual appointment in May 2018. The data was analysed using Excel 2010.

Results: 240 patients had virtual appointments in one month. 121 $(50.4 \%)$ were via telephone and 119 (49.6\%) via patient letter. 34 (14.1\%) patients had multiple virtual/telephone appointments. 129 (54\%) were carried out by consultants, $78(32 \%)$ by nurses and $33(14 \%)$ by registrars. $37 \%$ had rheumatoid arthritis. 32 (13\%) appointments lead to a prescription. Virtual appointments produced 44 referrals, including 18 to another specialty, 16 to physiotherapy, and the rest to hand therapy or podiatry. Most patients had a F2F appointment before and after their virtual appointment in May 2018, 1 patient had died before having a second face to face appointment and $13(5 \%)$ were discharged from their virtual appointment.

\begin{tabular}{lc}
\hline & $\begin{array}{c}\text { Mean Number of Days between } \\
\text { appointments }\end{array}$ \\
\hline $\begin{array}{l}\text { From } 1^{\text {st }} \text { Face to Face to Virtual } \\
\text { Appointment }\end{array}$ & 44 \\
From Virtual Appointment to $2^{\text {nd }}$ Face to & 53 \\
Face & 94 \\
From $1^{\text {st }}$ Face to Face to $2^{\text {nd }}$ Face to Face & 94 \\
\hline
\end{tabular}

Abstract AB1421HPR Table 1. The mean number of days between each type of appointment.

Conclusion: Consultants undertook the bulk of virtual clinics, and these appointments resulted in the majority of referrals and prescriptions. Virtua appointments reduce the waiting times for contact with a healthcare professional between appointments. Many patients had several virtua appointments between face to face appointments and this cohort may benefit from more scrutiny. Current technology already imporves communication and leads to significant changes in patient care without requiring F2F appointments. Internet based and app based interaction should face the same scrutiny.

Disclosure of Interests: None declared

DOI: 10.1136/annrheumdis-2019-eular.2688

\section{AB1422-HPR MODERATE AND HIGH ADHERENCE TO A DISEASE MANAGEMENT MODEL IN PATIENTS WITH RHEUMATOID ARTHRITIS IMPROVES CLINICAL RESULTS IN A BIG UNICENTRIC COHORT}

Laura Villarreal ${ }^{1}$, Fernando Rodriguez ${ }^{2}$, Michael Cabrera ${ }^{3}$, Pedro Santos-Moreno ${ }^{4}$ ${ }^{1}$ Biomab - Center for rheumatoid arthritis, Psychology, Bogoț. Colombia; ${ }^{2}$ Biomab - Center for rheumatoid arthritis, Patient program coordinator, Bogot. Colombia; ${ }^{3}$ Biomab - Center for rheumatoid arthritis, EHR administration, Bogot Colombia; ${ }^{4}$ Biomab, Rheumatology, Bogot Colombia

Background: Rheumatoid arthritis (RA) is an inflammatory, chronic disease of unknown etiology. Usually it leads to deformity and destruction of joints through the erosion of cartilage and bone. Over $90 \%$ of patients with RA report to suffer symptoms in hands and joints, swelling, loss of motion, muscle weakness among others. These symptoms affect all aspects in a patients life. Therefore, management of a patient with RA should not only include evaluate outcomes related to the rheumatology specialty, on the contrary, aspects such as physical disability, nutrition, mental health, among others should be taken into account. Centers of excellence in rheumatoid arthritis have proposed a multidisciplinary model of care with an initial diagnosis, treatment prescription and follow-up with a rheumatologist, periodic consultations with a physiatrist, psychologist, physiotherapist, occupational therapy nutrition, and, a patient focused program. With a multidisciplinary model of care the patient is seen as a whole, and the expectation is to achieve the best results in the management of RA.

Objectives: The aim of this research was to define adherence/attendance to a multidisciplinary model of care for patients with RA that attend to a RA specialized center in Colombia.

Methods: We implemented the center of excellence model program proposed by REAL-PANLAR group in 2015 (3). In order to define adherence to the multiapproach model the authors performed an informal expert consensus to propose a method to measure adherence to the model. The authors proposed three levels of adherence. We proposed three levels of adherence as follows: High adherence: For rheumatology patients had to attend between 6 and 12 consultations in one year. For physical therapy, physiatry, psychology, occupational therapy and nutrition patients had to attend to 3 or more consultations during one year per each specialty. Moderate adherence: For rheumatology patients had to attend between 3 and 5 consultations in one year. For physical therapy, physiatry, psychology, occupational therapy and nutrition patients had to attend between 2 or 4 consultations during one year per each specialty. Low adherence: For rheumatology patients had to attend between 1 and 2 consultations. For physical therapy, physiatry, psychology, occupational therapy and nutrition patients achieved only 1 consultation or less during one year per each specialty. We performed a descriptive analysis and compared the level of adherence and disease activity.

Results: During 2018 we reviewed the medical charts of 6851 patients diagnosed with rheumatoid arthritis; $82 \%$ were female and $18 \%$ were male. Mean age was 59 years 13 years old. Regarding disease activity mean DAS28 was 2.690 .84 . Most of patients that were considered as Moderate or High adherent achieve remission or LDA. See table 1. Levels of Adherence in Patients with RA

\begin{tabular}{lcccccc}
\hline DAS & LOW & \multicolumn{3}{c}{ MODER } & & HIGH \\
& ADH & & ADH & & ADH & \\
\cline { 2 - 7 } & $\mathrm{n}$ & $\%$ & $\mathrm{n}$ & $\%$ & $\mathrm{n}$ & $\%$ \\
\hline REMISION & 245 & $4 \%$ & 2090 & $31 \%$ & 1495 & $22 \%$ \\
LDA & 121 & $2 \%$ & 874 & $13 \%$ & 589 & $9 \%$ \\
MDA & 193 & $3 \%$ & 699 & $10 \%$ & 465 & $7 \%$ \\
SDA & 27 & $0.4 \%$ & 31 & $0.5 \%$ & 22 & $0 \%$ \\
\hline
\end{tabular}

Conclusion: This is an initial approach in order to evaluate patients adherence and attendance to a new implemented multidisciplinary disease management model of attention for patients with RA in Colombia. Our descriptive study demonstrated that patients with moderate or high adherence can achieve better clinical outcomes compared to those who arent adherent to the model.

Disclosure of Interests: Laura Villarreal: None declared, Fernando Rodriguez: None declared, Michael Cabrera: None declared, Pedro Santos-Moreno Grant/research support from: Dr Santos has received research grants from Janssen, Abbvie and UCB, Speakers bureau: $\mathrm{Dr}$ Santos has received speaker fees from Sanofi, Lilly, Bristol, Pfizer, Abbvie, Janssen and UCB

DOI: 10.1136/annrheumdis-2019-eular.6459

\section{AB1423-HPR ELICITING THE AGENDA OF PATIENTS WITH MUSCULOSKELETAL DISORDERS; THE PHYSIOTHERAPIST-PATIENT INTERACTION}

Sercan Yilli ${ }^{1}$, Serkan Kalmaz ${ }^{1}$, Faruk Altay ${ }^{2}$, Elif Turgut ${ }^{2} .{ }^{1}$ Hacettepe University, Ankara, Turkey, ${ }^{2}$ Hacettepe University, Department of Physiotherapy and Rehabilitation, Ankara, Turkey

Background: Eliciting main concerns via interviewing is important for patientcentered care and for planning individualized rehabilitation program $(1,2)$ Recently, it has been shown that clinicians often fail to elicit the patients agenda and, when they do, they interrupt the patients discourse (3). However, the prevalence of agenda setting in physiotherapist as a health care provider and the physiotherapist-patient interaction remains relatively unexplored.

Objectives: The aim of this study was to describe agenda elicitation in rehabilitation, to determine the frequency of encounters in which physiotherapists elicited the patients with musculoskeletal disorders agenda, the proportion and timing of interrupted answers.

Methods: An audio-recording analysis of 52 clinical encounters recorded during first physiotherapist-patient interaction were performed. The elicitation of the patient agenda characteristics as the time to interruption or to complete statement were analyzed.

Results: Physiotherapists elicited the patients agenda in all $(96.1 \%)$ clinical encounters. Interestingly, in those encounters in which physiotherapists elicited patient concerns, the clinician interrupted the patient after a median of 15 seconds (interquartile range 6 to 22 seconds). In the un interrupted encounters in which physiotherapists elicited patient concerns, the patients with musculoskeletal disorders was state their agenda in 2414.5 seconds.

Conclusion: Physiotherapist emphasize to elicit the patients agenda, however, they interrupt expression very sooner. Eventually, the failure to elici the patients agenda inhibits the physiotherapists-patient communication and this would lead to failure to plan rehabilitation program based on the needs of each patient.

\section{REFERENCES}

[1] Dyche L, Swiderski DJJogim. The effect of physician solicitation approaches on ability to identify patient concerns. 2005;20(3):267-70 